Texture of Crystalline Solids, 1981, Vol. 4, pp. 143-151

0390-7951/81/0403-0143\$06.50/0

(c) 1981 Gordon and Breach, Science Publishers, Inc.

Printed in the United States of America

\title{
A NEW LIBRARY PROGRAM FOR GENERATING AUGMENTED JACOBI POLYNOMIALS FOR TEXTURE CALCULATIONS
}

\author{
J. I. OHSUGI \\ Salesian Polytechnic, \\ Suginami-ku, Tokyo 167, Japan \\ and \\ T. FUJII \\ National Research Institute for Metals, \\ Meguro-ku, Tokyo 153, Japan \\ (Received November 6, 1980)
}

\begin{abstract}
A new library program for generating augmented Jacobi polynomials for texture analysis is presented. By using this program, the spatial orientation distribution maps for the three-dimensional texture analysis can be produced.
\end{abstract}

\section{INTRODUCTION}

In the three-dimensional texture analysis, numerical values of polynomials should be calculated by using generalized Legendre polynomials or augmented Jacobi polynomials.

These polynomials denoted $\mathrm{P}_{\ell}^{\mathrm{mn}}(\Phi)$ by $B$ unge ${ }^{1}$ and $\mathrm{Z}_{\ell \mathrm{mn}}(\xi)$ by $R_{0}{ }^{2}$ are identical with each other except for their normalization constants and, in some cases, sign.

However, the polynomials $\mathrm{Z}_{\ell m n}(\xi)$ (we define hereafter, according to the notations used by Roe) have up to now been calculated in the form of Fourier series ${ }^{3-6}$; the Fourier coefficients in tabular form have already been provided by Morris et al. ${ }^{3}, 4$

If the numerical values of polynomials expanded in Fourier series are adopted in the computer program for ODF analysis, the following disadvantages occur:

(1) A computer with a large memory is required to store resident data.

(2) Errors of numerical values in a table of Fourier coefficients cause errors in the calculation of the polynomials $\mathrm{Z}_{\ell \mathrm{mn}}(\xi)$.

To avoid these disadvantages, two methods have been considered feasible to calculate the numerical values of polynomials $\mathrm{Z}_{\ell m n}(\xi)$ : The first one is deducing $\mathrm{Z}_{\ell m n}(\xi)$ from $a$ 
recurrence relation. The second is deducing $\mathrm{z}_{\ell \mathrm{mn}}(\xi)$ from the hypergeometric series directly. Iiang et al. ${ }^{7}$ suggested in the recent report the method of generating the polynomials $\mathrm{z}_{\ell \mathrm{mn}}(\xi)$ by the first method. To apply this method, however, initial values for each $l, m, n$ must be given previously, which would inevitably lead to the increase in program size, required memory, and processing time.

The purposes of this report are to produce a library program for generating $\mathrm{Z}_{\ell \mathrm{mn}}(\xi)$ based on the second method; and by using this program to work out the orientation distribution maps for the rolled texture of b.c.c. metals, as an example, by use of a small computer (NEAC-3100).

DEFINITION OF $\mathrm{z}_{\ell \mathrm{mn}}(\xi)$ AND ITS GENERATION

The augmented Jacobi polynomials is defined by $R_{0}{ }^{2}$ as follows:

$$
Z_{\ell m n}(\xi)=N_{\ell m n} t^{(m-n) / 2}(1-t)^{(m+n) / 2} f(t)
$$

where

$$
\begin{aligned}
& t=\frac{1-\xi}{2} ; \\
& N_{\ell m n}=\left[\frac{2 \ell+1}{2} \cdot \frac{(\ell+m) !(\ell-n) !}{(\ell-m) !(\ell+n) !}\right]^{\frac{3}{2}} \cdot \frac{1}{(m-n) !} ; \\
& f(t)={ }_{2} F_{1}(\alpha, \beta ; \gamma ; t) .
\end{aligned}
$$

${ }_{2} F_{1}(\alpha, \beta ; \gamma ; t)$ is Gauss' hypergeometric series (see Appendix). The ${ }_{2} F_{1}$ is generated easily as shown in Figure 1 , where $k$ and $\varepsilon$ are pointer and allowance error, respectively. The symbol $(:=)$ shows that the calculation result on the right hand side should be substituted in the variable on the left hand side. The polynomials $\mathrm{Z}_{\ell \mathrm{mn}}(\xi)$ are generated by using the ${ }_{2} \mathrm{~F}_{1}$ as shown in Figure 2. According to Figure 1 and Figure 2, a library program for generating augmented Jacobi polynomials was formed in FORTRAN for small computer (NEAC-3100) and in BASIC for personal computer (PC-8001).

We verified the library program by calculating normalized Legendre polynomials $\mathrm{z}_{\ell 00}(\xi)$ and normalized associated Legendre polynomials $\mathrm{z}_{\ell \mathrm{mo}}(\xi)$, and proved by numerical integration that the polynomials $z_{\ell m n}(\xi)$ generated by this program satisfy the orthogonal relation ${ }^{2}$

$$
\int_{-1}^{1} z_{\ell m n}(\xi) z_{\ell \cdot m n}(\xi) d \xi=\delta_{\ell, \ell} .
$$




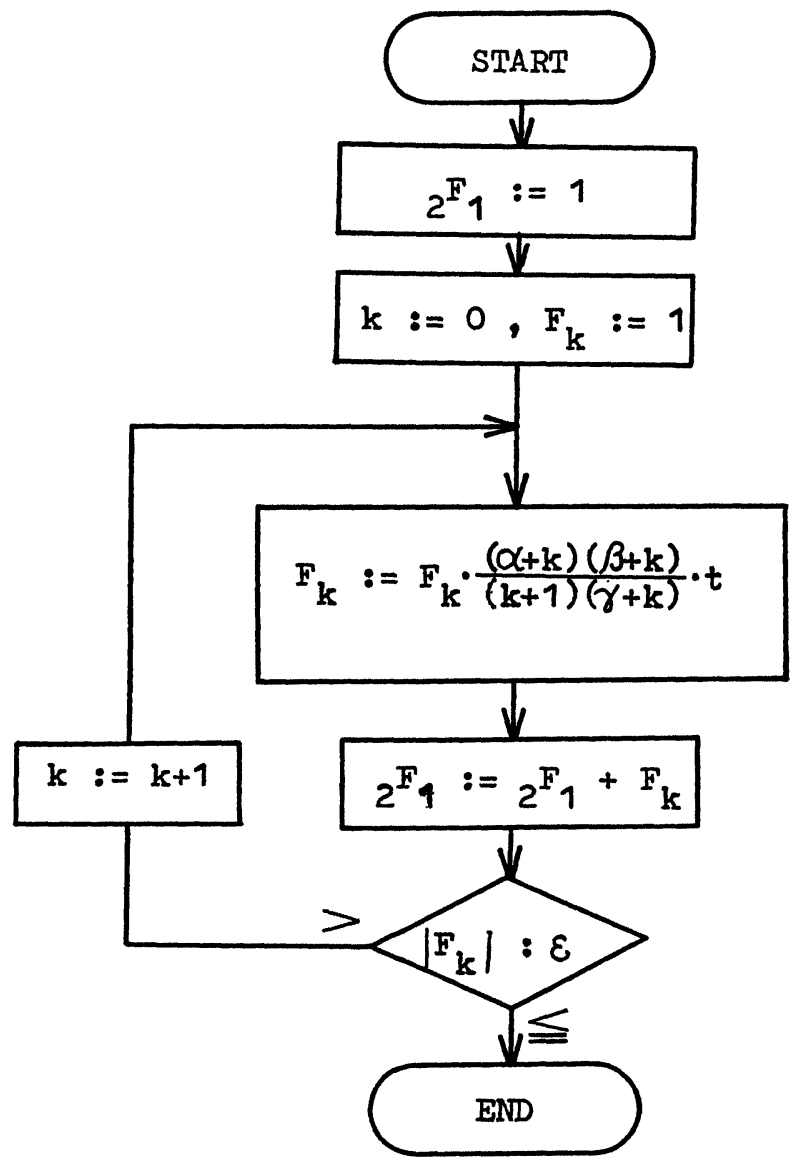

Figure 1. Algorithm to generate ${ }_{2} F_{1}(\alpha, \beta ; \gamma ; t)$.

PRODUCTION OF SPATIAL ORIENTATION DISTRIBUTION OF CRYSTALLITES

In setting up our library program by using the coefficients of ODF given by $\mathrm{Hu},{ }^{8}$ the spatial orientation distribution of crystallites in the as-cold-rolled phosphorus steel sheet can be obtained, as shown in Figure 3. The picture at constant ph $\left(\phi=45^{\circ}\right)$ is almost the same as that obtained by $\mathrm{Hu}$.

Figure 4 shows the spatial orientation distribution of crystalites in the as-rolled molybdenum TZM-sheet at constant phi $\left(\phi=45^{\circ}\right)$, which is produced by using coefficients of ODF determined from our library of $z_{l m n}(\xi)$. For the spatial orientation distribution map, texture data from X-ray pole figure were used in the form of one of the incomplete pole figures obtained only by the Schulz back reflection technique. 


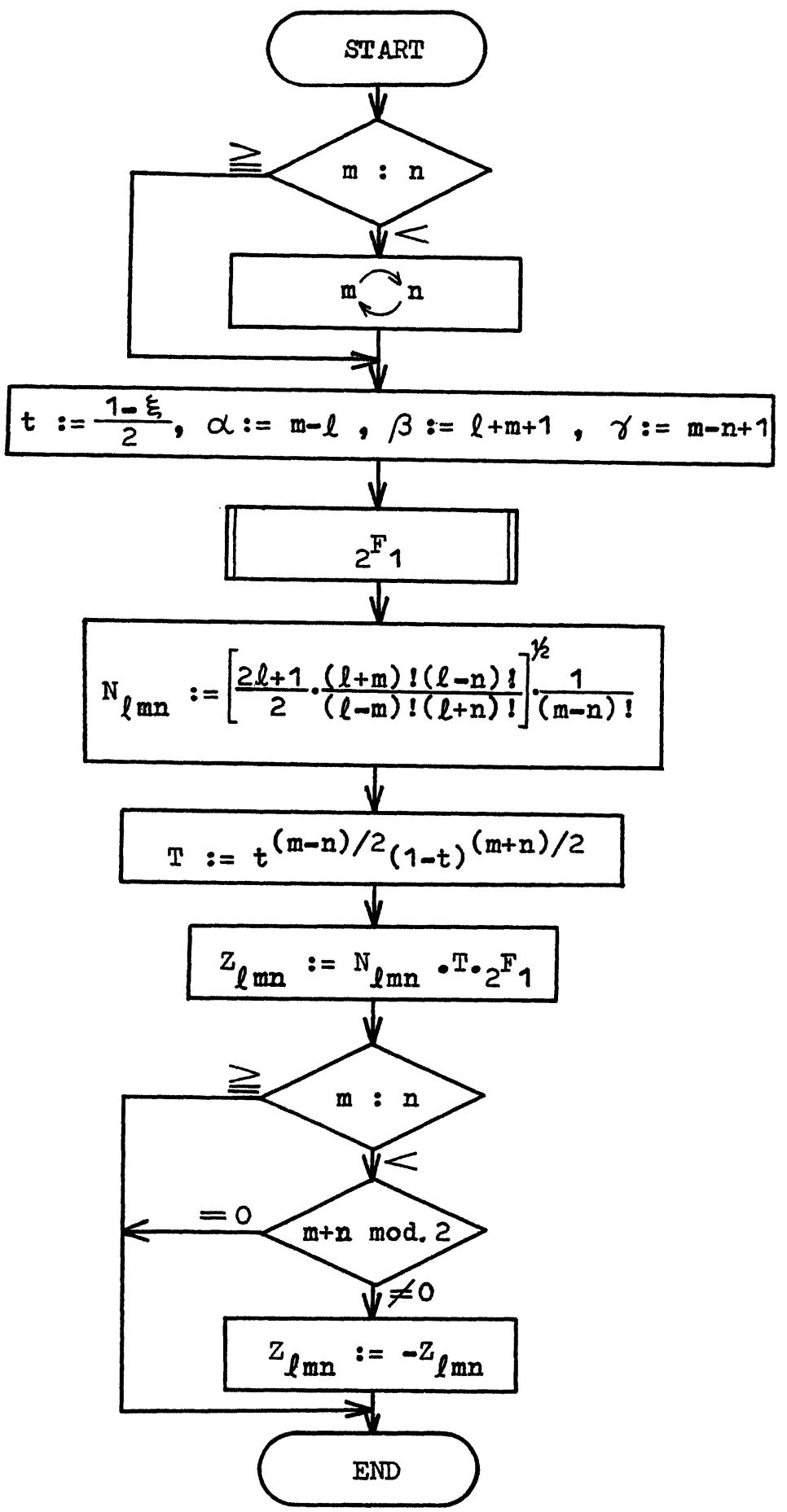
Figure 2. Algorithm to generate $z_{\ell m n}(\xi), ~$
$m$ and $n$ means interchanging 
*** +IGURE UF UUF FUK PHI $=43.0 * * *$

MAX $=0.43127$ MIN $=-1.230 \angle 3 \quad$ PSI

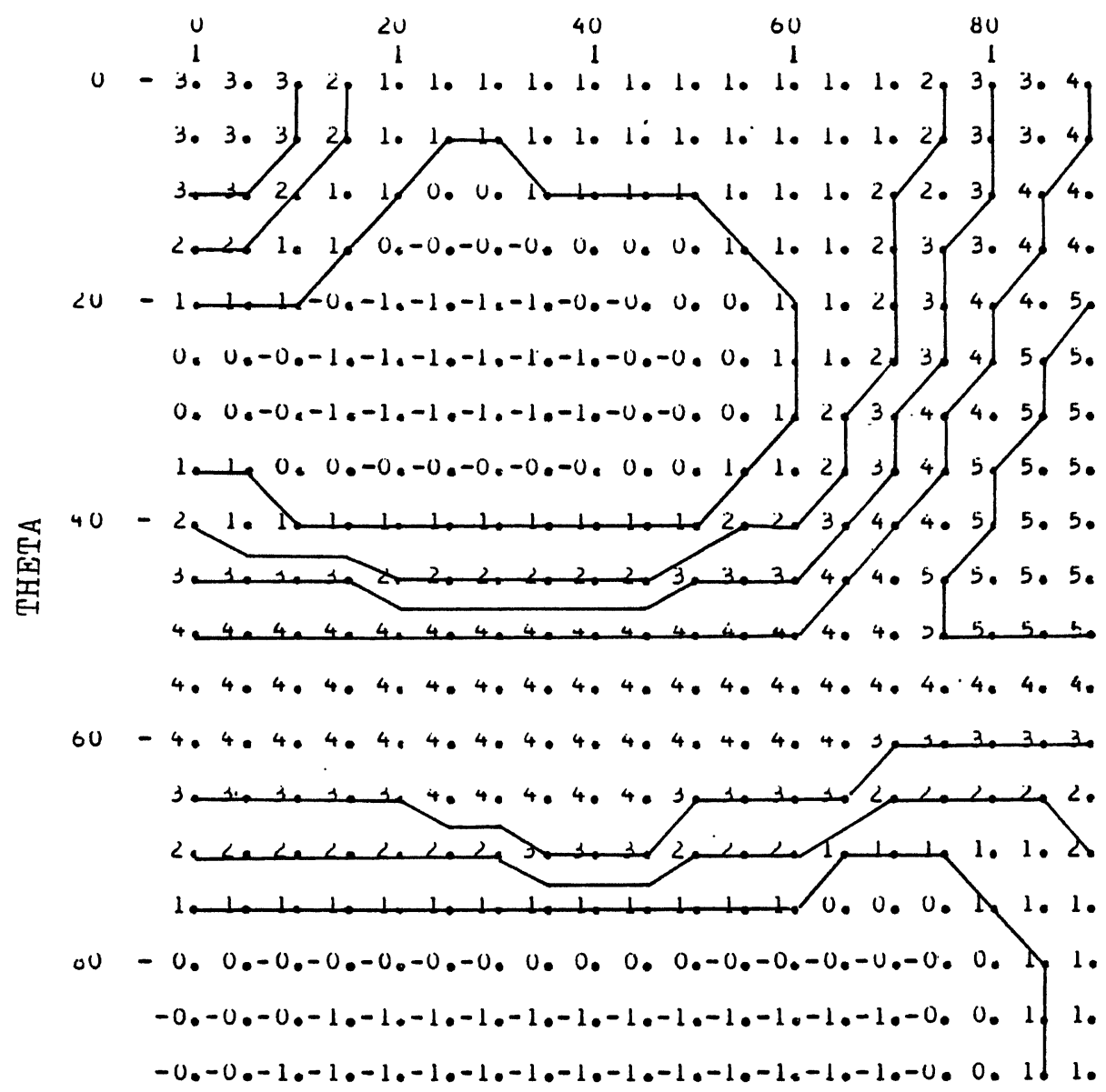

KANUJM INTENSIIY $=1$

Figure 3. Spatial orientation distribution map of crystallite in the as-cold-rolled phosphorus-sheet, psi $(\psi)$ vs theta $(\theta)$ at constant phi $\left(\phi=45^{\circ}\right)$ section, produced by using coefficients of ODF given by Hu. . 
*** FIGLFE UF UUF FOR PHI $=45.0 * * *$

MAX $=9.98500$ MIN $=-1.30170 \quad$ PSI

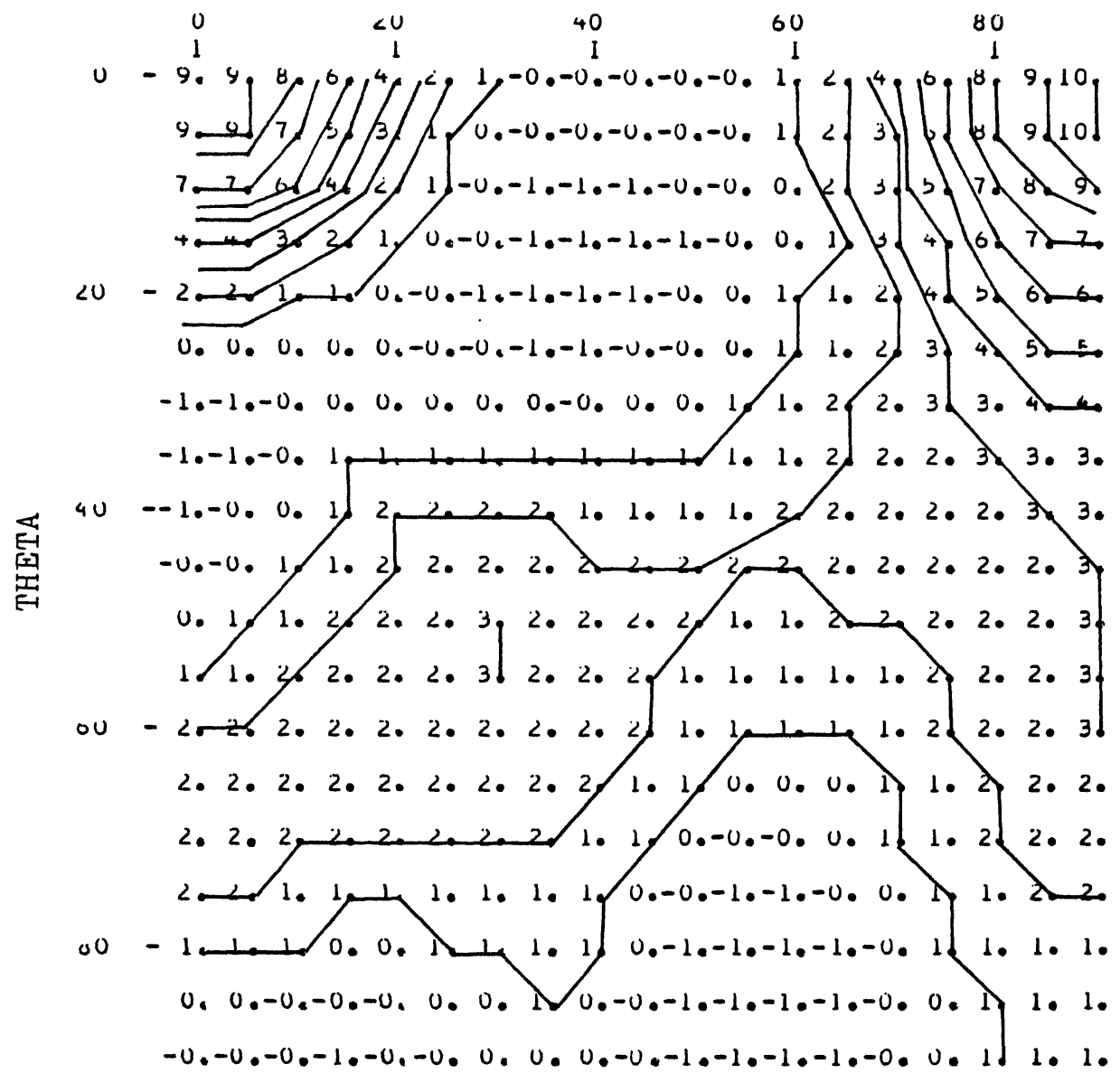

RAINUJM INTENSITY $=1$

Figure 4. Spatial orientation distribution map of crystallites in the as-rolled molybdenum TZM-sheet, psi $(\psi)$ vs theta $(\theta)$ at constant phi $\left(\phi=45^{\circ}\right)$ section, produced by using coefficients of ODF determined from our library.

\section{CONCLUSION}

A new library program for generating augmented Jacobi polynomials for three-dimensional texture analysis has been carried out for the purpose of obtaining the spatial orientation distribution of crystallites by the use of a small computer (NEAC-3100) or a personal computer (PC-8001). The details of the program, the required memory capacity and the 
accuracy in the computer calculation will be presented in a forthcoming paper.

\section{ACKNOWLEDGEMENTS}

The authors would like to thank Dr. I. Nishida of the Metal Physics Division of the National Research Institute for Metals for his helpful discussion, and J. Y. Kido of the Electrical Engineering Department of the Salesian Polytechnic for his cordial assistance with the computer program.

\section{APPENDIX}

The series ${ }_{2} F_{2}(\alpha, \beta ; \gamma ; t)$ is known as Gauss' hypergeometric series. This series has been generalized by the introduction of parameters $p$ and $q$ as follows: ${ }^{10}$

$$
{ }_{p} F_{q}\left(\alpha_{i} ; \gamma_{j} ; t\right)=\sum_{n=1}^{\infty} \frac{\left(\alpha_{1}\right)_{n}\left(\alpha_{2}\right)_{n} \cdots\left(\alpha_{p}\right)_{n}}{\left(\gamma_{1}\right)_{n}} \frac{t^{n}}{\left(\gamma_{2}\right)_{n} \cdots\left(\gamma_{q}\right)_{n}}
$$

where

$$
(x)_{n}=\frac{\Gamma(x+n)}{\Gamma(x)}
$$

and $\Gamma(x)$ is Gamma function. When $x$ is integer,

$$
(\mathrm{x})_{\mathrm{n}}=\mathrm{x}(\mathrm{x}+1) \ldots(\mathrm{x}+\mathrm{n}-1) \text {. }
$$

The series $\mathrm{pFq}$ is known as Pochhammer's generalized hypergeometric series. This series is terminated by the negative integer $\alpha s$, in which case it is useful in the physical sciences. In the case of $\mathrm{p}=\mathrm{q}=1$, the series is written as ${ }_{1} F_{1}(\alpha ; \gamma, t)$ and called "Kummer's confluent hypergeometric series." 11

Moreover, Kummer's confluent hypergeometric series leads to Laguerre, associated Laguerre and Hermite polynomials. Also, Gauss' hypergeometric series leads to Legendre, associated Legendre, Jacobi, Gegenbauer and Tchebycheff polynomials.12,13 It is, therefore, very important to deduce the polynomials $\mathrm{pFq}$.

The series $\mathrm{pF}_{\mathrm{q}}$ is generated easily according to the algorithm as shown in Figure 5 .

\section{REFERENCES}

1. H. J. Bunge, Mathematische Methoden der Texturanalyse, AkademieVerlag, Berlin, 1969, Chap. 11, pp. 215-261.

2. R. J. Roe, J. Appl. Phys., 36, 2024 (1965).

3. P. R. Morris and A. J. Heckler, Advances in X-Ray Analysis, 11, 454 (1968).

4. P. R. Morris and A. J. Heckler, Trans. TMS-AIME, 245, 1877 (1969).

5. J. Pospiech and J. Jura, Kristall und Technik, 10, 783 (1975).

6. P. R. Morris, Texture Cryst. Sol., 2, 57 (1976).

7. Z. D. Liang, J. Z. Xu and F. Wang, Texture Cryst. Sol., 4, 93 (1980).

8. H. Hu, Texture Cryst. Sol., 4, 13 (1980). 


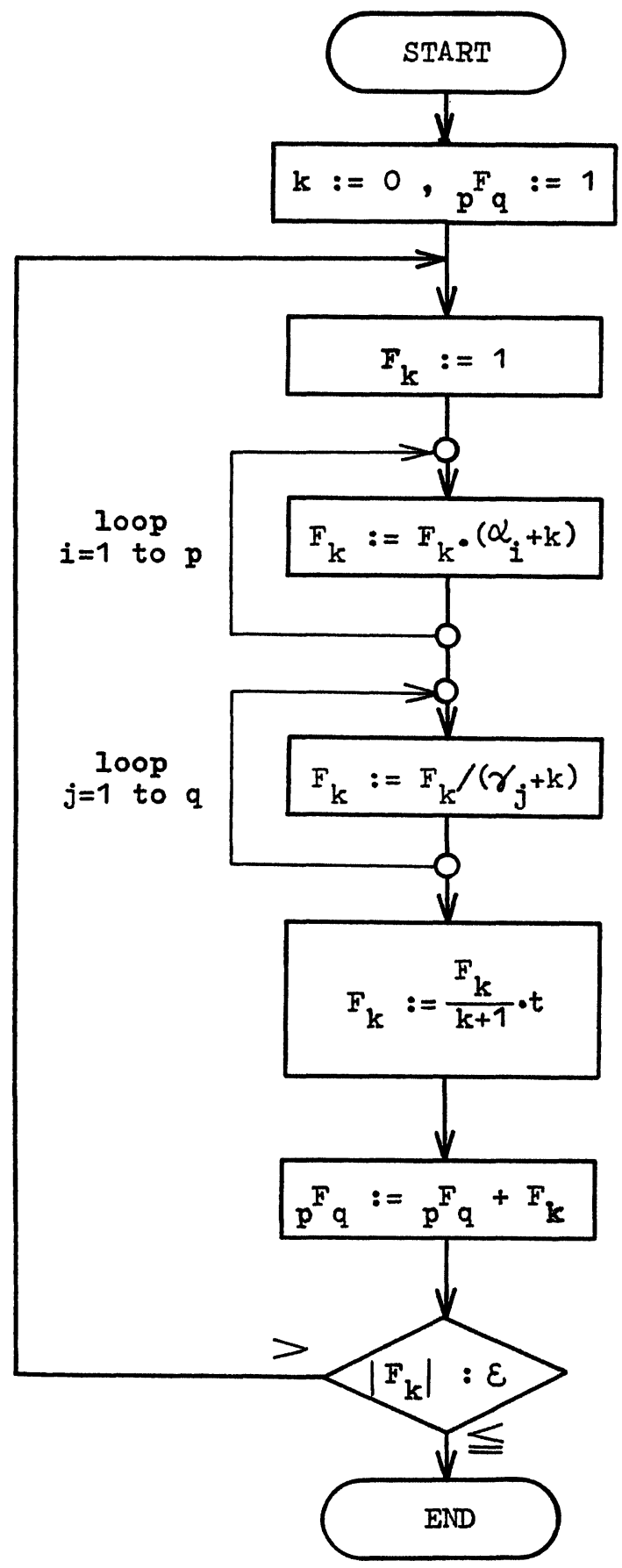

Figure 5. Algorithm to generate $\mathrm{pF}_{\mathrm{q}}\left(\alpha_{i} ; \gamma_{j} ; t\right)$. 
9. A. Erdélyi, Higher Transcendental Functions (Bateman Manuscript Project), McGraw-Hill Book Co., New York, 1953, Vol. 1, Chap. 2, pp. 56-117.

10. Ibid., Vol. 1, Chap. 4, pp. 182-199.

11. Ibid., Vol. 1, Chap. 6, pp. 248-293.

12. Ibid., Vol. 2, Chap. 10, pp. 153-228.

13. Ibid., Vol. 3, Chap. 19, pp. 245-279. 